\title{
EKF-Based Actuator Fault Detection and Diagnosis Method for Tilt-Rotor Unmanned Aerial Vehicles
}

\author{
Jiaxin Gao, ${ }^{1}$ Qian Zhang $\mathbb{D}^{2}$ and Jiyang Chen $\mathbb{D}^{1}$ \\ ${ }^{1}$ School of Instrumentation and Optoelectronic Engineering, Beihang University, Beijing 100191, China \\ ${ }^{2}$ School of Aeronautic Science and Engineering, Beihang University, Beijing 100191, China \\ Correspondence should be addressed to Qian Zhang; victoryqian175@163.com
}

Received 23 April 2020; Revised 8 July 2020; Accepted 15 July 2020; Published 3 August 2020

Academic Editor: Laurent Dewasme

Copyright (c) 2020 Jiaxin Gao et al. This is an open access article distributed under the Creative Commons Attribution License, which permits unrestricted use, distribution, and reproduction in any medium, provided the original work is properly cited.

Flight safety is of vital importance for tilt-rotor unmanned aerial vehicles (UAVs), which can take off and land vertically as well as cruise at high speed, especially in different kinds of complex environment. As being the executor of the flight control, the actuator failure will directly affect the controllability of the tilt-rotor UAV, and it has high probability of causing fatal personal injury and financial loss. However, due to the limitation of weight and cost, small UAVs cannot be equipped with redundant actuators. Therefore, there is an urgent need of fault detection and diagnosis method for the actuators. In this paper, an actuator fault detection and diagnosis (FDD) method based on the extended Kalman filter (EKF) and multiple-model adaptive estimation (MMAE) is proposed. The actuator deflections are added to the state vector and estimated using EKF. The fault diagnosis algorithm of MMAE could assign a conditional probability to each faulty actuator according to the residual of EKF and diagnose the fault. This paper is structured as follows: first, the structure and model of tilt-rotor UAV actuator are established. Then, EKF observers are introduced to estimate the state vector and to calculate residual sequences caused by different faulty actuators. The residuals from EKFs are used by fault diagnosis algorithm to assign a conditional probability to each failure condition, and fault type can be diagnosed according to the probabilities. The FDD method is verified by simulations, and the results demonstrate that the FDD algorithm could accurately and efficiently diagnose actuator fault without any additional sensor.

\section{Introduction}

Tilt-rotor UAV, equipped with tilt-rotors, is a new type of UAV which can take off and land vertically on any fixed ground as well as cruise at high speed [1-3]. The tilt-rotor UAVs are being increasingly used in civil and military applications, such as the search and rescue operations, remote sensing, geographic studies, weather monitoring, and various military applications [4-8]. It is one of the important development directions of UAV in the future.

The tilt-rotor UAV is similar to the fixed wing in the horizontal flight stage. Its attitudes are controlled by the aerodynamic lift acting on the deflected control surfaces which are powered by the actuators of the flight controller $[9,10]$. During the mission, the environment is complex and changeable, and actuator failure is inevitable. Generally, the small and low-cost UAVs are equipped with only one set of actuators, and there is no redundant actuator backup and no sensors that measure actuator deflections. Actuator failure will directly affect the flight controllability of the tilt-rotor UAV, which can cause fatal personal injury and property loss. Therefore, the actuator failure of the tilt-rotor UAV has the most serious impact on flight safety. So, in order to detect various kinds of faults and to maximum ensure flight safety, it is essential to design an actuator FDD method in the flight control system $[7,11]$.

Several model-based techniques have been used for FDD [12-15]. A linear fault-tolerant control method was investigated by Zhang et al. in [16], which used an iterative learning observer to estimate both stuck actuator deflection and state vector. This method can be adapted to many stuck actuators.

An actuator fault detection and accommodation method for linear MIMO system was presented to handle loss of 
actuator effectiveness in [17]. This method separated the stochastic system into two subsystems. One of them is no-fault system, whose states were estimated by a reduced-order Kalman filter. The other is affected by the faults. In [18], Amoozgar and Chamseddine proposed a quadrotor helicopter actuator FDD system. A two-stage Kalman filter was used to isolate and estimate possible faults, which were modeled as losses in the control effectiveness of rotors, in each actuator. A sensor fault detection and diagnosis system for small autonomous helicopters was presented in [19]. The system used a Kalman filter observer, obtained from input-output experimental data, to detect fault by evaluating any significant change in the behavior of the vehicle. The system is implemented with real flight data, and the results demonstrate that "hard" and "soft" failures can be detected by the fault detection system, and the detection results are better and more robust when compared to the obtained using ARX linear observers. In [20], a fault detection and diagnosis strategy based on neural network and fuzzy system is proposed to detect sensor and actuator faults of UAV. The strategy was verified with a flight control system of UAV, and the results showed that this strategy could successfully detect the sensor faults.

Multiple-model adaptive estimation is a common method to detect actuator or sensor fault [21]. It is composed of multiple Kalman filters, and each of them corresponds to the system state under a specific fault. According to the residual of each Kalman filter output, the hypothesis testing algorithm sets the conditional probability of each fault. As long as there are a certain number of Kalman filters to estimate the expected fault, the MMAE method can be applied in practice. However, enough Kalman filters are required to cover the possible faults, so the calculation of this method is very large, and the fault that can be isolated is limited. Particularly for the stuck fault, the filters have high efficiency only near the preset working state. In addition, the Kalman filter used in the MMAE method is mostly suitable for describing linear differential equation, while for most practical applications, the dynamics of tilt-rotor UAV is mostly described by nonlinear differential equation.

In this paper, an actuator fault detection and diagnosis method is proposed to monitor the health status of the tiltrotor UAV actuators. An extended Kalman filter is introduced in the FDD method, which increases the actuator deflection to the state vector. And the control input matrix and dynamics matrix of the EKF are modified for nonlinear estimation of the actuator deflection and other fault parameters. Only one filter will be used to monitor the health status of one actuator, which greatly reduces the number of filters used to monitor actuator failure. Furthermore, the MMAE method is combined with the extended Kalman filter to calculate the fault conditional probability online according to the residual and the state error covariance matrix of each EKF. Then the faulty actuator can be isolated. In this case, the proposed method can accurately and efficiently detect the actuator faults, even if the actuator is stuck near the trim position. The effectiveness of the proposed method is implemented and evaluated by simulations, and the simulation results show that the actuator FDD strategy could diagnose the actuator faults successfully with high levels of accuracy and efficiency without any need to add additional sensors to measure actuator deflections or to change the flight controller.

The article is organized as follows. In the next section, the structure of the tilt-rotor UAV is presented, and the actuator model is established. In Section 3, the actuator FDD method based on the EKF and MMAE is introduced, and the theoretical basis of extended Kalman filter is derived step by step. The numerical simulations as well as their results are conducted and analyzed in Section 4. Finally, conclusions are drawn in Section 5.

\section{Structure and Model of the Tilt-Rotor UAV Actuator}

2.1. Structure of the Tilt-Rotor UAV. The research object of this paper is a tilt-rotor UAV, which adopts blended wing body configuration and has two control surfaces, as shown in Figure 1. One is left aileron, and the other is right aileron. Left/right ailerons are completely independent, which means that ailerons could rotate in one direction together or move up and down independently. This structure can generate some pitching motion by using the synchronous motion of aileron or some rolling motion by using the differential of aileron. The physical parameters of the tilt-rotor UAV are described in Table 1.

The coordinate systems involved in this paper are configured as follows. The tilt-rotor UAV chooses the eastnorth-up geographic coordinate system as the navigation system ( $n$-frame, $O-X_{n} Y_{n} Z_{n}$ ). The axis direction of the body coordinate system ( $b$-frame, $O-X_{b} Y_{b} Z_{b}$ ) is defined as follows: $X$-axis is pointing to the forward side of the aircraft, $Y$-axis is along the right side of the aircraft, $Z$-axis is pointing to the vertical side, and $X, Y$, and $Z$-axes are in a right-handed coordinate system.

2.2. Aircraft Nonlinear Dynamics. During the flight, the aircraft makes various maneuvers by changing its attitude, which requires applying aerodynamic torque to the body. The aerodynamic torque is only related to the aerodynamic effect on the control surfaces of the tilt-rotor UAV such as the left and right ailerons. The relationship between angular velocity and the torques applied to the aircraft can be written as follows:

$$
\left[\begin{array}{c}
\dot{p} \\
\dot{q} \\
\dot{r}
\end{array}\right]=\left(\mathbf{I}^{b}\right)^{-1}\left(\left[\begin{array}{c}
L \\
M \\
N
\end{array}\right]^{b}-\left[\begin{array}{c}
p \\
q \\
r
\end{array}\right] \times I^{b}\left[\begin{array}{l}
p \\
q \\
r
\end{array}\right]\right),
$$

where $p$ is the roll rate of the UAV. $q$ is the pitch rate of the UAV. $r$ is the yaw rate of the UAV. $\mathbf{I}^{b}$ is the body-fixed inertia matrix of the UAV. $L, M, N$ represent the rolling torque, pitching torque, and yawing torque of UAV, respectively.

In this paper, the aerodynamic torques of the small tilt-rotor unmanned aerial vehicle are modeled as follows [10]: 


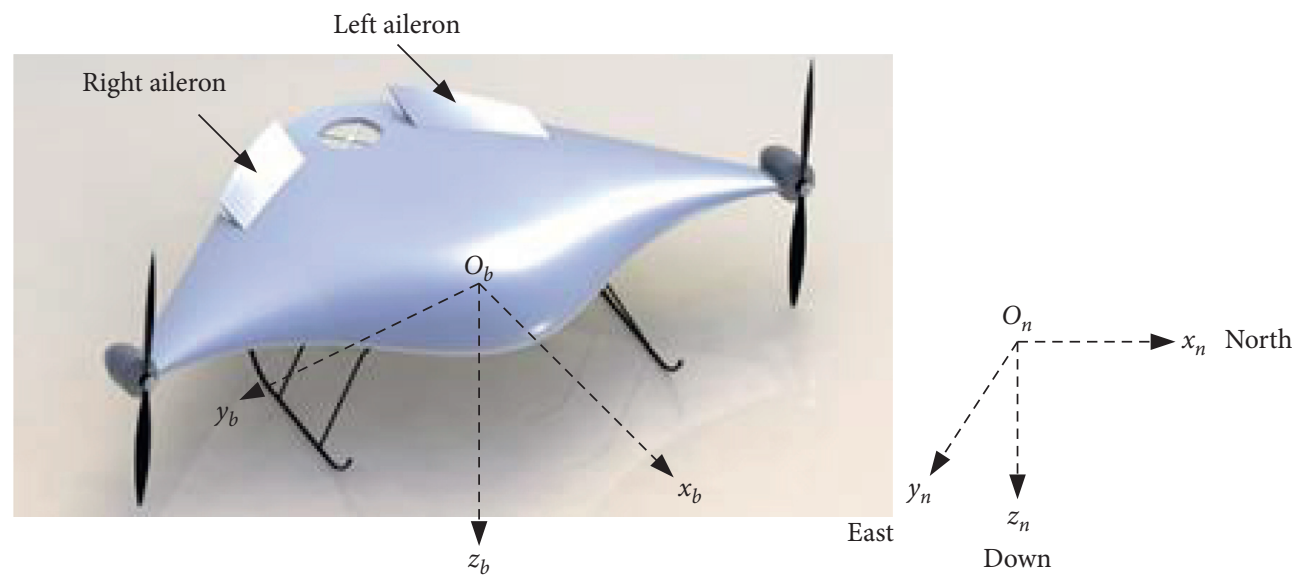

FIgURE 1: Structure of the tilt-rotor UAV.

TABle 1: Physical parameters of the tilt-rotor UAV.

\begin{tabular}{lc}
\hline Parameters & Value \\
\hline Weight & $65 \mathrm{~kg}$ \\
Wing area & $4.01 \mathrm{~m}^{2}$ \\
Mean aerodynamic wing chord & $1.27 \mathrm{~m}$ \\
Wing span & $3.15 \mathrm{~m}$ \\
Moment of inertia $I_{x x}$ & $53.31 \mathrm{~kg} \mathrm{~m}^{2}$ \\
Moment of inertia $I_{y y}$ & $18.38 \mathrm{~kg} \mathrm{~m}^{2}$ \\
Moment of inertia $I_{z z}$ & $69.47 \mathrm{~kg} \mathrm{~m}^{2}$ \\
\hline
\end{tabular}

$$
\begin{aligned}
L & =\bar{q} S b C_{L}\left(\delta_{a l}, \delta_{a r}, p, r, \beta\right), \\
M & =\bar{q} S \bar{c} C_{M}\left(\delta_{a l}, \delta_{a r}, \alpha, q,\right) . \\
N & =\bar{q} S b C_{N}\left(\delta_{a l}, \delta_{a r}, r, \beta\right),
\end{aligned}
$$

where $\bar{q}$ is dynamic pressure; $\bar{q}=(1 / 2) \rho V^{2} . S$ is the wing reference area of UAV. $b$ is the wing span length and $\bar{c}$ is the mean aerodynamic wing chord. $C_{L}, C_{M}, C_{N}$ represent the correlation aerodynamic coefficient, respectively. The UAV's left aileron is $\delta_{a l}$; its right aileron is $\delta_{a r} . \alpha$ is the angle of attack. $\beta$ is the sideslip angle.

The inertia matrix is

$$
I=\left[\begin{array}{ccc}
I_{x x} & 0 & I_{x z} \\
0 & I_{y y} & 0 \\
I_{z x} & 0 & I_{z z}
\end{array}\right],
$$

where the aircraft is symmetrical about the $X O Z$ plane, so $I_{x y}$ and $I_{y z}$ are assumed to be 0 according to the shape of the UAV. $I_{x x}$ is the moment of inertia about the $x$-axis. $I_{y y}$ is the moment of inertia about the $y$-axis. $I_{z z}$ is the moment of inertia about the $z$-axis. $I_{x z}$ and $I_{z x}$ are the product of inertia.

2.3. Model of the Tilt-Rotor UAV Actuator. There are two types of faults in UAV actuators [22]; one is the complete failure state that does not respond to the control command completely; for example, the control surface is stuck or swung. The other is the partial failure state that can respond to the control command but cannot achieve the expected control effect. For example, when the control surface is damaged, the control gain of the channel will be reduced, which cannot achieve the expected control effect. Among them, the stuck fault of actuator will make the UAV unable to respond to the command of flight control system, which is the most dangerous to the flight safety.

When the actuator is stuck or swung at a certain position, it can be regarded as the expected input control command of the actuator is disconnected and replaced by a wrong control command. The fault model of UAV actuator is shown in Figure 2.

According to Figure 2, the real input of the actuator can be written as follows:

$$
u_{i}(t)=\delta_{i}(t)+\sigma_{A i}\left(\bar{\delta}_{i}(t)-\delta_{i}(t)\right) .
$$

When the actuator fails, the unknown deflection variables can be written as

$$
\bar{\delta}(t)=\left[\bar{\delta}_{1}(t) \bar{\delta}_{2}(t), \ldots, \bar{\delta}_{n}(t)\right]^{T},
$$

where $u_{i}$ is the real input of the $i$ th actuator of UAV. $\delta_{i}$ is the expected deflection of the $i$ th actuator without failure. $\bar{\delta}_{i}$ is the deflection of the $i$ th actuator under fault condition. $\sigma_{A i}$ is the fault sign of the $i$ th actuator.

$$
\sigma_{A i}= \begin{cases}1, & \text { when } i^{\text {th }} \text { actuator fault } \\ 0, & \text { otherwise. }\end{cases}
$$

The deflection of the $i$ th actuator under fault condition $\bar{\delta}_{i}$ will be estimated by its corresponding extended Kalman filter.

\section{Actuator Fault Detection and Diagnosis Method}

3.1. Multiple-Model Adaptive Estimation Method. The MMAE method uses a set of parallel Kalman filters for fault detection and diagnosis, and each Kalman filter corresponds to one fault state. When a predefined fault state occurs, the diagnosis algorithm calculates the fault probability of each actuator according to the residual error and the state error covariance matrix of each Kalman filter. Its basic working principle is shown in Figure 3. 


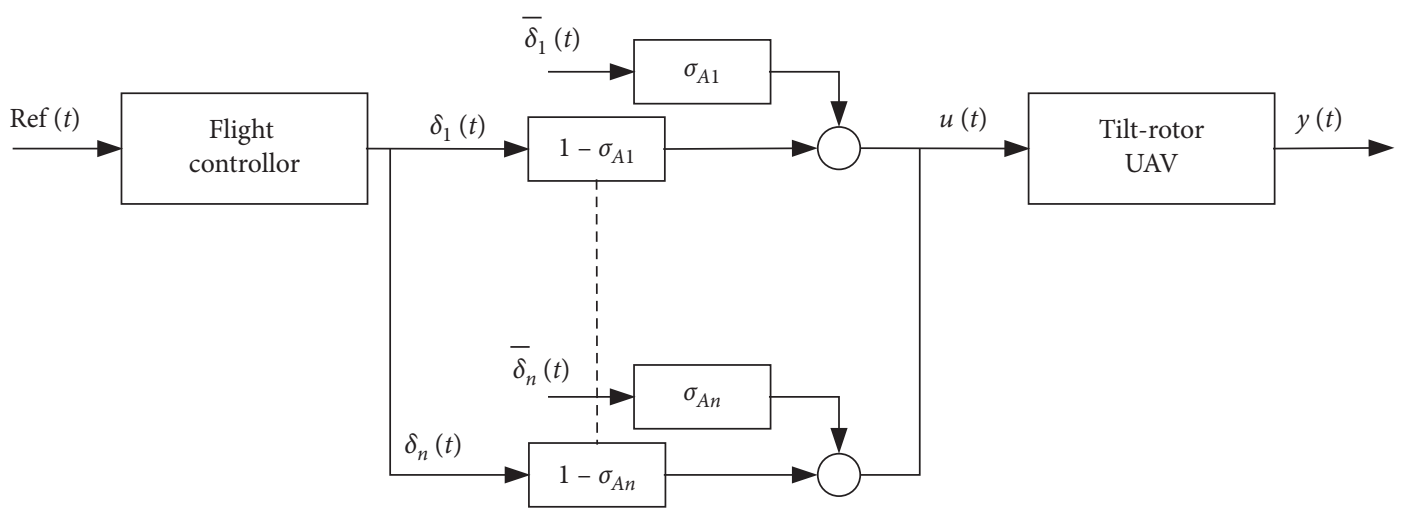

FIgURe 2: Actuator fault model of the tilt-rotor UAV.

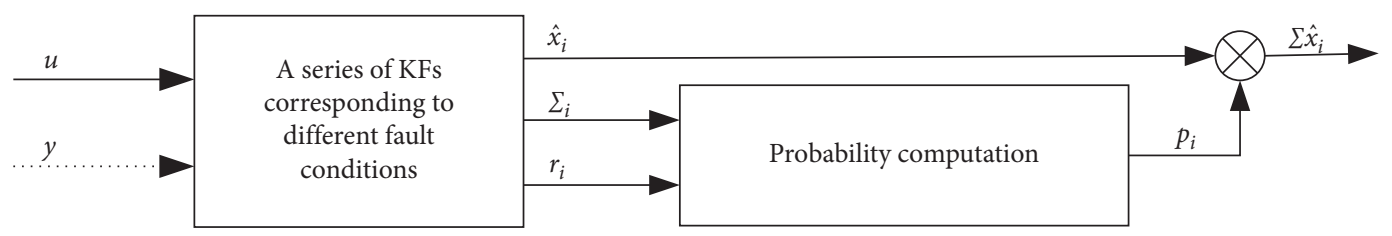

FIgURE 3: The MMAE method.

However, the MMAE method has some limitations in practical application. First of all, the number of Kalman filters used by MMAE method must cover all possible faults, which directly leads to the huge computation cost of the MMAE method. Secondly, the detection and diagnosis algorithm has higher efficiency only when it works near the predefined working state since each Kalman filter of the MMAE method is corresponding to a predefined fault state. When the actual fault of the actuator is different from the predefined fault state, the MMAE method may not get accurate fault diagnosis results. Finally, when the actuator is stuck at any nonzero position, the performance of the Kalman filter will be affected since the residuals of the filter will be biased because of the ignorance of the actuator deflection, which will lead to error fault diagnosis result and inaccurate state variable estimation. The tilt-rotor UAV is impossible to effectively apply the fault diagnosis results to reconstruct flight control system or control the UAV to avoid threat.

3.2. FDD Method Based on the EKF and MMAE. The FDD method used in this paper combines the extended Kalman filter with the MMAE method and uses the extended Kalman filter to replace the linear Kalman filter in the MMAE method. At the same time, the actuator deflection is chosen as a part of state variables of the filter to estimate the system state and fault parameters. Therefore, compared with the MMAE method, the extended Kalman filter of the FDD method can be applied to all possible fault positions of actuator, not only to a predefined fault position. By using only one extended Kalman filter, the whole fault conditions of one actuator can be monitored, which greatly reduces the number of filters used for actuator fault diagnosis and decreases the computing load. The working principle of the
FDD method based on EKF and MMAE is shown in Figure 4.

In the FDD method, a model is defined to describe the $i$ th actuator fault. The $i$ th column of the control input matrix is modified to 0 . Then, the state variable is extended by increasing the actuator deflection of the $i$ th actuator $\bar{\delta}_{i}$ to estimate the $i$ th actuator deflection $\widehat{\bar{\delta}}_{i}$ all the time. In addition, the dynamics matrix is expanded, and the aircraft aerodynamics model corresponding to the ith filter is changed by adding the elements of the control input matrix of the $i$ th column. In this way, the control input from the $i$ th actuator of the control system will be completely ignored, but the faulty actuator deflection in the state vector will be continuously estimated to modify the UAV aerodynamics model of the $i$ th filter, which minimizes the residuals of the filter and makes the filter always correspond to the fault that occurred. Hence, even if the actuator is stuck at a nonzero position, the FDD algorithm can efficiently diagnose the actuator fault and estimate the system state and the deflection of faulty actuator.

3.3. Extended Kalman Filter Design. The continuous nonlinear differential equations describing the dynamic characteristics of the tilt-rotor UAV in EKF can be written as [23]

$$
\begin{aligned}
& \dot{X}=\mathbf{f}(\mathbf{X}, \mathbf{u})+\mathbf{w}, \\
& \mathbf{Z}=\mathbf{h}(\mathbf{X})+\mathbf{v},
\end{aligned}
$$

where $\mathbf{f}(\mathbf{X}, \mathbf{u})$ is the nonlinear function of the state variable and the control input variable. $\mathbf{X}$ is the state vector. $\mathbf{u}$ is the control input variable, and $\mathbf{w}$ is the zero mean random noise vector. $\mathbf{Z}$ is the measurement vector, and $\mathbf{v}$ is the measurement noise vector, which is used to describe measurement noise of each sensor. 


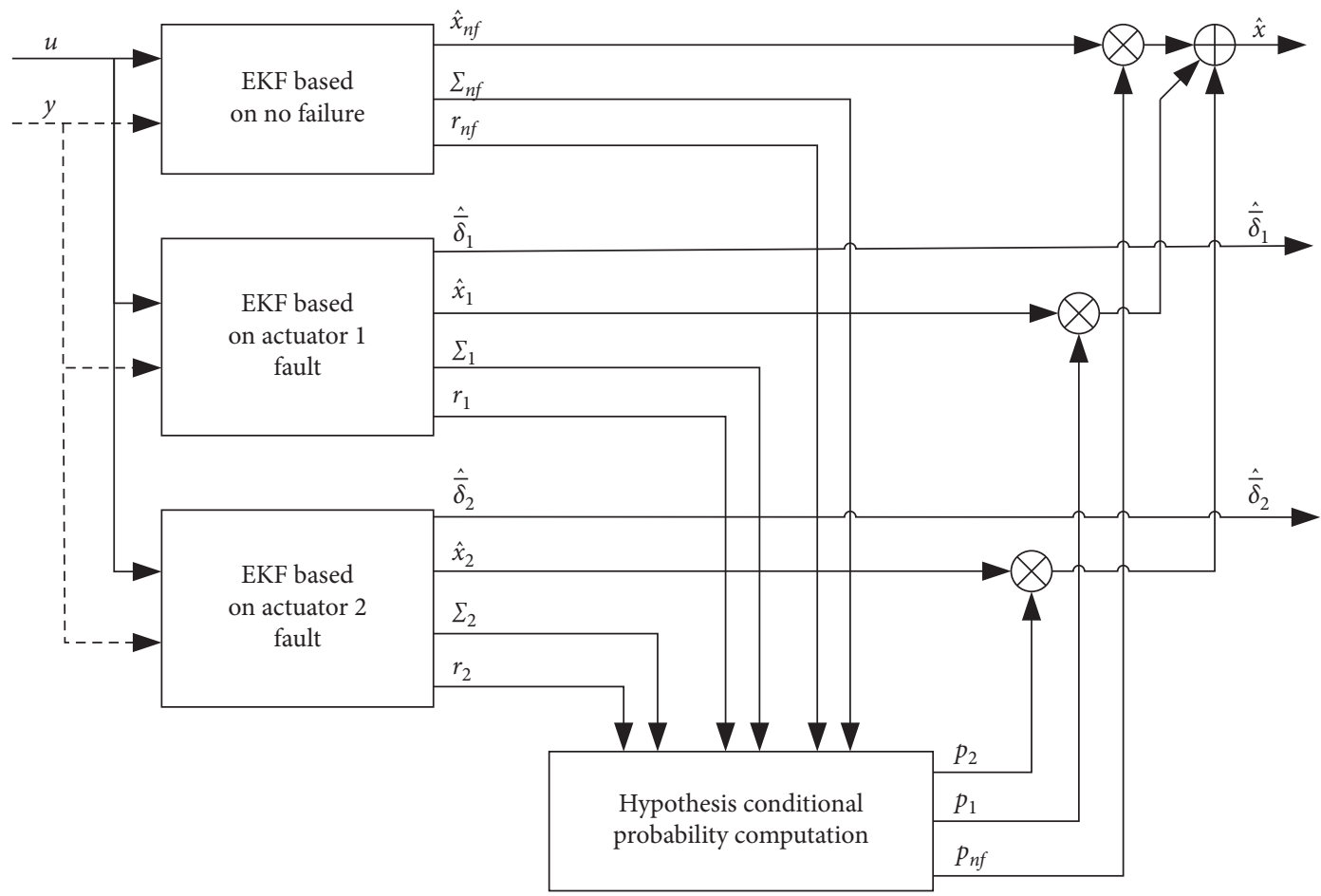

FIGURE 4: Working principle of the FDD method based on EKF and MMAE.

For this kind of continuous nonlinear differential equation, firstly, the equation is linearized at the working point; then it is discretized by the Euler integral method. After discretization, (7) and (8) can be expressed as follows:

$$
\begin{aligned}
\mathbf{X}_{k+1} & =\boldsymbol{\varphi}_{k+1, k} \mathbf{X}_{k}+\mathbf{G}_{k} \mathbf{U}_{k}+\Gamma_{k} \mathbf{W}_{k}, \\
\mathbf{Z}_{k} & =\mathbf{H}_{k} \mathbf{X}_{k}+\mathbf{V}_{k},
\end{aligned}
$$

where the discrete transformation matrix $\varphi_{k+1, k} \approx \mathbf{I}+\mathbf{F}(k) T_{s}$, $\mathbf{I}$ is an $n$ identity matrix. $T_{s}$ is the sampling time of the system. $\mathbf{G}_{k}$ is the control input matrix. $\Gamma_{k}$ is the system noise transformation matrix. $\mathbf{F}(k)$ is the dynamic matrix of continuous system which can be calculated as follows:

$$
\begin{aligned}
& \mathbf{F}(k)=\frac{\partial \mathbf{f}(\mathbf{X}, \mathbf{u})}{\partial \mathbf{X}}, \\
& \mathbf{F}(k)=\left[\begin{array}{ccc}
\frac{C_{l p} S b^{2}}{2 V_{a} I_{x x}} q_{*} & 0 & \frac{C_{l r} S b^{2}}{2 V_{a} I_{x x}} q_{*} \\
\frac{C_{m p} S c_{A}^{2}}{2 V_{a} I_{y y}} q_{*} & \frac{C_{m q} S c_{A}^{2}}{2 V_{a} I_{y y}} q_{*} & \frac{C_{m r} S c_{A}^{2}}{2 V_{a} I_{y y}} q_{*} \\
\frac{C_{n p} S b^{2}}{2 V_{a} I_{z z}} q_{*} & 0 & \frac{C_{n r} S b^{2}}{2 V_{a} I_{z z}} q_{*}
\end{array}\right], \\
& \mathbf{G}(k)=\left[\begin{array}{cc}
0 & \frac{C_{l \delta_{d}} S b}{I_{x x}} q_{*} \\
\frac{C_{m \delta_{t}} S c_{A}}{I_{y y}} q_{*} & 0 \\
0 & \frac{C_{n \delta_{d}} S b}{I_{z z}} q
\end{array}\right]\left[\begin{array}{cc}
\frac{1}{2} & \frac{1}{2} \\
\frac{1}{2} & -\frac{1}{2}
\end{array}\right]=\left[\begin{array}{cc}
\frac{C_{l \delta_{d}} S b}{2 I_{x x}} q_{*} & -\frac{C_{l \delta_{d}} S b}{2 I_{x x}} q_{*} \\
\frac{C_{m \delta_{t}} S c_{A}}{2 I_{y y}} q_{*} & \frac{C_{m \delta_{t}} S c_{A}}{2 I_{y y}} q_{*} \\
\frac{C_{n \delta_{d}} S b}{2 I_{z z}} q_{*} & -\frac{C_{n \delta_{d}} S b}{2 I_{z z}} q_{*}
\end{array}\right] \text {, } \\
& \mathbf{H}(k)=\left[\begin{array}{lll}
1 & 0 & 0 \\
0 & 1 & 0 \\
0 & 0 & 1
\end{array}\right] \text {, }
\end{aligned}
$$


where $q_{*}$ is the aerodynamic pressure $q_{*}=(1 / 2) \rho V_{a}^{2} \cdot V_{a}$ is UAV's airspeed. $\rho$ is the air density. $S$ is the wing area, $b$ is the wing span, and $c_{A}$ is the average chord length.

$\mathbf{W}_{k}$ is discrete system random noise vector, which is used to describe the model uncertainty. $\mathbf{V}_{k}$ is discrete measurement random noise vector, which is used to describe measurement noise of each sensor. $\mathbf{W}_{k}$ and $\mathbf{V}_{k}$ satisfy the following equation:

$$
\begin{aligned}
E\left[\mathbf{W}_{k}\right] & =0, \operatorname{Cov}\left[\mathbf{W}_{k}, \mathbf{W}_{j}\right]=E\left[\mathbf{W}_{k} \mathbf{W}_{j}^{T}\right]=\mathbf{Q}_{k} \delta_{k j}, \\
E\left[\mathbf{V}_{k}\right] & =0, \operatorname{Cov}\left[\mathbf{V}_{k}, \mathbf{V}_{j}\right]=E\left[\mathbf{V}_{k} \mathbf{V}_{j}^{T}\right]=\mathbf{R}_{k} \delta_{k j},
\end{aligned}
$$

where $\delta_{k j}$ is the Kronecker delta function.

For reducing the computing load of the EKF, the variables most related to the aircraft state are the only variables selected as the state vector of the FDD filter. The state vector of the FDD filter is defined as $\mathbf{X}=[p, q, r]^{T}$, and the measurement vector of the FDD filter is defined as $\mathbf{Z}=[p, q, r]^{T}$. The control surface of the tilt-rotor UAV is two ailerons, so the control vector $\mathbf{u}$ is defined as $\mathbf{u}=\left[\delta_{r}, \delta_{l}\right]^{T}$.

Then, the equations used in FDD filters can be described as follows [24]:

$$
\left\{\begin{array}{l}
\widehat{X}_{k}=\left[\mathbf{I}-\mathbf{K}_{k} \mathbf{H}_{k}\right] \boldsymbol{\varphi}_{k, k-1} \widehat{X}_{k, k-1}+\mathbf{K}_{k} \mathbf{Z}_{k}, \\
\mathbf{P}_{k, k-1}=\boldsymbol{\varphi}_{k, k-1} \mathbf{P}_{k-1} \boldsymbol{\varphi}_{k, k-1}^{T}+\Gamma_{k-1} \mathbf{Q}_{k-1} \Gamma_{k-1}^{T}, \\
\mathbf{P}_{k}=\left[\mathbf{I}-\mathbf{K}_{k} \mathbf{H}_{k}\right] \mathbf{P}_{k, k-1}, \\
\mathbf{K}_{k}=\mathbf{P}_{k, k-1} \mathrm{H}_{k}^{T}\left[\mathbf{H}_{k} \mathbf{P}_{k, k-1} \mathbf{H}_{k}^{T}+\mathbf{R}_{k}\right]^{-1}
\end{array}\right.
$$

In this case, the continuous transformation matrix and control input matrix of the no-fault extended Kalman filter can be expressed as

$$
\begin{aligned}
& \mathbf{F}_{n f}(k)=\left[\begin{array}{lcc}
\frac{C_{l p} S b^{2}}{2 V_{a} I_{x x}} q_{*} & 0 & \frac{C_{l r} S b^{2}}{2 V_{a} I_{x x}} q_{*} \\
\frac{C_{m p} S c_{A}^{2}}{2 V_{a} I_{y y}} q_{*} & \frac{C_{m q} S c_{A}^{2}}{2 V_{a} I_{y y}} q_{*} & \frac{C_{m r} S c_{A}^{2}}{2 V_{a} I_{y y}} q_{*} \\
\frac{C_{n p} S b^{2}}{2 V_{a} I_{z z}} q_{*} & 0 & \frac{C_{n r} S b^{2}}{2 V_{a} I_{z z}} q_{*}
\end{array}\right], \\
& \mathbf{G}_{n f}(k)=\left[\begin{array}{ll}
\frac{C_{l \delta_{d}} S b}{2 I_{x x}} q_{*} & -\frac{C_{l \delta_{d}} S b}{2 I_{x x}} q_{*} \\
\frac{C_{m \delta_{t}} S c_{A}}{2 I_{y y}} q_{*} & \frac{C_{m \delta_{t}} S c_{A}}{2 I_{y y}} q_{*} \\
\frac{C_{n \delta_{d}} S b}{2 I_{z z}} q_{*} & -\frac{C_{n \delta_{d}} S b}{2 I_{z z}} q_{*}
\end{array}\right],
\end{aligned}
$$

where $Q_{*}=(1 / 2) \rho V^{2} S$. The discrete transformation matrix of no-fault filter can be written as $\varphi_{n f, k}=\mathbf{I}+\mathbf{F}_{n f}(k) T_{s}$, and the discrete control input matrix is $\mathbf{G}_{n f, k}=\mathbf{G}_{n f}(k) T_{s}$.
Taking the right aileron fault as an example, a filter is designed to monitor the fault status of the right aileron. The state vector of the filter corresponding to the right aileron is extended by adding the deflection of the faulty right aileron $\bar{\delta}_{r}$ in the state vector to monitor the occurrence of the right aileron fault. The state vector $\mathbf{X}_{\delta_{r}}$ can be written as

$$
X_{\delta_{r}}=\left[\begin{array}{c}
X \\
\bar{\delta}_{r}
\end{array}\right]=\left[p, q, r, \bar{\delta}_{r}\right]^{T} .
$$

The extended state vector $\mathbf{X}_{\delta_{r}}$ satisfies the following equations:

$$
\begin{aligned}
\mathbf{X}_{\delta_{r}}(k+1) & =\mathbf{f}_{x_{\delta_{r}}}\left(\mathbf{X}_{\delta_{r}}(k), \boldsymbol{\delta}(k)\right)+\mathbf{W}(k), \\
\mathbf{Z}_{\delta_{r}}(k) & =\mathbf{h}\left(\mathbf{X}_{\delta_{r}}(k)\right)+\mathbf{V}(k) .
\end{aligned}
$$

Equation (15) is linearized at the working point, and the linearized system can be expressed as follows:

$$
\begin{aligned}
{\left[\begin{array}{c}
\mathbf{X}(k+1) \\
\bar{\delta}_{r}(k+1)
\end{array}\right] } & =\boldsymbol{\varphi}_{\delta_{r}, k}\left[\begin{array}{l}
\mathbf{X}(k) \\
\bar{\delta}_{r}(k)
\end{array}\right]+\mathbf{G}_{\delta_{r}, k} \boldsymbol{\delta}(k), \\
\mathbf{Z}(k) & =\mathbf{H}_{\delta_{r}}(k)\left[\begin{array}{c}
\mathbf{X}(k) \\
\bar{\delta}_{r}(k)
\end{array}\right],
\end{aligned}
$$

where the discrete transformation matrix of the right aileron fault filter can be calculated as $\varphi_{\delta_{r}, k}=\mathbf{I}+\mathbf{F}_{\delta_{r}}(k) T_{s}$, and the discrete control input matrix of the right aileron fault filter can be calculated as $\mathbf{G}_{\delta_{r}, k}=\mathbf{G}_{\delta_{r}}(k) T_{s}$, where $\mathbf{F}_{\delta_{r}}(k)$ and $\mathbf{G}_{\delta_{r}}(k)$ can be obtained as follows:

$$
\mathbf{F}_{\delta_{r}}(k)=\left[\begin{array}{llll}
\frac{C_{l p} S b^{2}}{2 V_{a} I_{x x}} q_{*} & 0 & \frac{C_{l r} S b^{2}}{2 V_{a} I_{x x}} q_{*} & \frac{C_{l \delta_{d}} S b}{2 I_{x x}} q_{*} \\
\frac{C_{m p} S c_{A}^{2}}{2 V_{a} I_{y y}} q_{*} & \frac{C_{m q} S c_{A}^{2}}{2 V_{a} I_{y y}} q_{*} & \frac{C_{m r} S c_{A}^{2}}{2 V_{a} I_{y y}} q_{*} & \frac{C_{m \delta_{t}} S c_{A}}{2 I_{y y}} q_{*} \\
\frac{C_{n p} S b^{2}}{2 V_{a} I_{z z}} q_{*} & 0 & \frac{C_{n r} S b^{2}}{2 V_{a} I_{z z}} q_{*} & \frac{C_{n \delta_{d}} S b}{2 I_{z z}} q_{*} \\
0 & 0 & 0 & 1
\end{array}\right],
$$

The measurement matrix of the right aileron fault filter can be expressed as follows. The extended Kalman filter for 
monitoring the left aileron fault can be designed in a similar way:

$$
\mathbf{H}_{\delta_{r}}(k)=\left[\begin{array}{ll}
\mathbf{H}(k) & 0
\end{array}\right] .
$$

3.4. Actuator Fault Diagnosis. The actuator fault diagnosis algorithm sets the conditional probability of each fault according to the residual and covariance matrix of state error of each extended Kalman filter. In order to determine which actuator is most likely to fail, the diagnosis algorithm needs to refer to the measured data. The most recently available measurement vector is $\mathbf{Z}_{k}$, and the final measurement sequence is defined as $\vec{Z}_{k}=\left\{\mathbf{Z}_{k}, \mathbf{Z}_{k-1}, \mathbf{Z}_{k-2}, \ldots, \mathbf{Z}_{0}\right\}$. The fault probability $p_{\delta_{i}}[k]$ can be expressed as the posterior conditional probability $p_{\delta_{i}}[k]=p\left(\delta_{i} \mid \vec{Z}_{k}\right)$, which means the probability of actuator failure $\delta_{i}$ under the condition of the last sequencing sequence $\vec{Z}_{k}$. According to Bayes theorem, the fault probability $p_{\delta_{i}}[k]$ can be written as

$$
p_{\delta_{i}}[k]=p\left(\delta_{i} \mid \vec{Z}_{k}\right)=\frac{p\left(\vec{Z}_{k} \mid \delta_{i}\right) \cdot p\left(\delta_{i}\right)}{p\left(\vec{Z}_{k}\right)} .
$$

According to the total probability theorem, probability $p\left(\vec{Z}_{k}\right)$ can be decomposed into

$$
\begin{gathered}
p\left(\vec{Z}_{k}\right)=p\left(\vec{Z}_{k} \mid \delta_{1}\right) \cdot p\left(\delta_{1}\right)+\cdots+p\left(\vec{Z}_{k} \mid \delta_{N}\right) \\
\cdot p\left(\delta_{N}\right)=\sum_{j=0}^{N} p\left(\vec{Z}_{k} \mid \delta_{j}\right) \cdot p\left(\delta_{j}\right) .
\end{gathered}
$$

Combining the above two equations, the fault probability $p_{\delta_{i}}[k]$ can be expressed as

$$
p_{\delta_{i}}[k]=p\left(\delta_{i} \mid \vec{Z}_{k}\right)=\frac{p\left(\vec{Z}_{k} \mid \delta_{i}\right) \cdot p\left(\delta_{i}\right)}{\sum_{j=0}^{N} p\left(\vec{Z}_{k} \mid \delta_{j}\right) \cdot p\left(\delta_{j}\right)} .
$$

To facilitate the recursive calculation of probability, the measurement sequence $\overrightarrow{\mathbf{Z}}_{k}$ can be written in the form of $\left\{\mathbf{z}_{k}, \vec{Z}_{k-1}\right\}$ :

$$
\begin{aligned}
p\left(\vec{Z}_{k} \mid \delta_{i}\right) & =p\left(\mathbf{Z}_{k}, \vec{Z}_{k-1} \mid \delta_{i}\right) \\
& =p\left(\mathbf{Z}_{k} \mid\left(\vec{Z}_{k-1}, \delta_{i}\right)\right) \cdot p\left(\vec{Z}_{k-1} \mid \delta_{i}\right) \\
& =p\left(\mathbf{Z}_{k} \mid\left(\delta_{i}, \vec{Z}_{k-1}\right)\right) \cdot p_{\delta_{i}}(k-1) .
\end{aligned}
$$

Using the result of (22) in (21), the fault probability $p_{\delta_{i}}[k]$ can be expressed as

$$
p_{\delta_{i}}(k)=p\left(\delta_{i} \mid \vec{Z}_{k}\right)=\frac{p\left(\mathbf{Z}_{k} \mid\left(\delta_{i}, \vec{Z}_{k-1}\right)\right) \cdot p_{\delta_{i}}(k-1) \cdot p\left(\delta_{i}\right)}{\sum_{j=0}^{N} p\left(\mathbf{Z}_{k} \mid\left(\delta_{j}, \vec{Z}_{k-1}\right)\right) \cdot p_{\delta_{j}}(k-1) \cdot p\left(\delta_{j}\right)} .
$$

In practice, the fault probability of each actuator is the same. Therefore, the same prior probability can be specified for all actuator faults $p\left(\delta_{j}\right)=1 / N$, where $N$ represents the number of filters including fault-free filter. According to Bayes theorem, the recursive form of the $i$ th actuator fault probability $p_{\delta_{i}}(k)$ can be written as follows:

$$
p_{\delta_{i}}(k)=p\left(\delta_{i} \mid \vec{Z}_{k}\right)=\frac{p\left(\mathbf{Z}=\mathbf{Z}_{k} \mid\left(\delta_{i}, \vec{Z}_{k-1}\right)\right) \cdot p_{\delta_{i}}(k-1)}{\sum_{j=0}^{N} p\left(\mathbf{Z}=\mathbf{Z}_{k} \mid\left(\delta_{j}, \vec{Z}_{k-1}\right)\right) \cdot p_{\delta_{j}}(k-1)},
$$

where the conditional probability $p\left(\mathbf{Z}=\mathbf{Z}_{k} \mid\left(\delta_{i}, \vec{Z}_{k-1}\right)\right)$ represents the probability that the system can obtain, the measurement data $\mathbf{Z}=\mathbf{Z}_{k}$ at time $k$ when the actuator fault $\delta_{i}$ exists, and the last measurement sequence of the system is $\vec{Z}_{k-1}$.

When the dynamic characteristics of the UAV change slowly, it can be assumed that the residual of the extended Kalman filter obeys the Gaussian distribution. For a single-input single-output system, the residual $r_{i}(k)$ represents the relative position between the estimated measurement data $H\left(\widehat{X}_{i}(k, k-1)\right)$ calculated by the $i$ th extended Kalman filter and the true measurement value $Z_{k}$, which is shown in Figure 5. Figure 6 shows the different shapes of Gaussian functions with several standard deviations.

The FDD system in this paper is a multi-input multioutput system. So, the conditional probability $p\left(\mathbf{Z}=\mathbf{Z}_{k} \mid\left(\delta_{i}, \vec{Z}_{k-1}\right)\right)$ can be calculated by a Gaussian probability density function with a bell-shaped curve [25], whose expression is as follows:

$$
\begin{aligned}
p\left(\mathbf{Z}=\mathbf{Z}_{k} \mid\left(\delta_{i}, \vec{Z}_{k-1}\right)\right) & =f\left(\mathbf{r}_{i} \mid\left(\delta_{i}, \vec{Z}_{k-1}\right)\right) \\
& =\lambda_{i}(k) e^{-r_{i}(k)^{T} \sum_{i}^{-1}(k) r_{i}(k) / 2},
\end{aligned}
$$

where $\lambda_{i}(k)=1 /\left((2 \pi)^{m / 2}\left|\Sigma_{i}(k)\right|\right)^{1 / 2}$, and $m$ is the dimension of the measurement vector. $\Sigma_{i}(k)$ is the covariance matrix value of the $i$ th extended Kalman filter residual and the shape of the Gaussian probability density function is determined by the covariance matrix $\Sigma_{i}(k) . r_{i}(k)$ is the residual of the $i$ th extended Kalman filter, which can be calculated by the following equation:

$$
\mathbf{r}_{i}(k)=\mathbf{Z}_{i}(k)-\mathbf{H}\left(\hat{X}_{i}(k, k-1)\right) .
$$

Therefore, when an actuator fault occurs, its corresponding extended Kalman filter will produce an estimation measurement vector $\widehat{Z}_{i}(k)=\mathbf{H}\left(\widehat{X}_{i}(k, k-1)\right)$, which is very close to the real measurement vector $\mathbf{Z}_{i}(k)$, and the residual $\mathbf{r}_{i}(k)$ is close to zero. This means that the extended Kalman filter matches this fault best, and the corresponding probability is maximum.

So, after calculating the conditional probability $p\left(\mathbf{Z}=\mathbf{Z}_{k} \mid\left(\delta_{i}, \mathbf{Z}_{k-1}\right)\right)$, it can be implied that whether every actuator is fault free or not by checking the fault probability $p_{\delta_{i}}(k)$ of each actuator at any time.

In addition, the method also can effectively estimate the state vector of the system with the state vector of each filter 


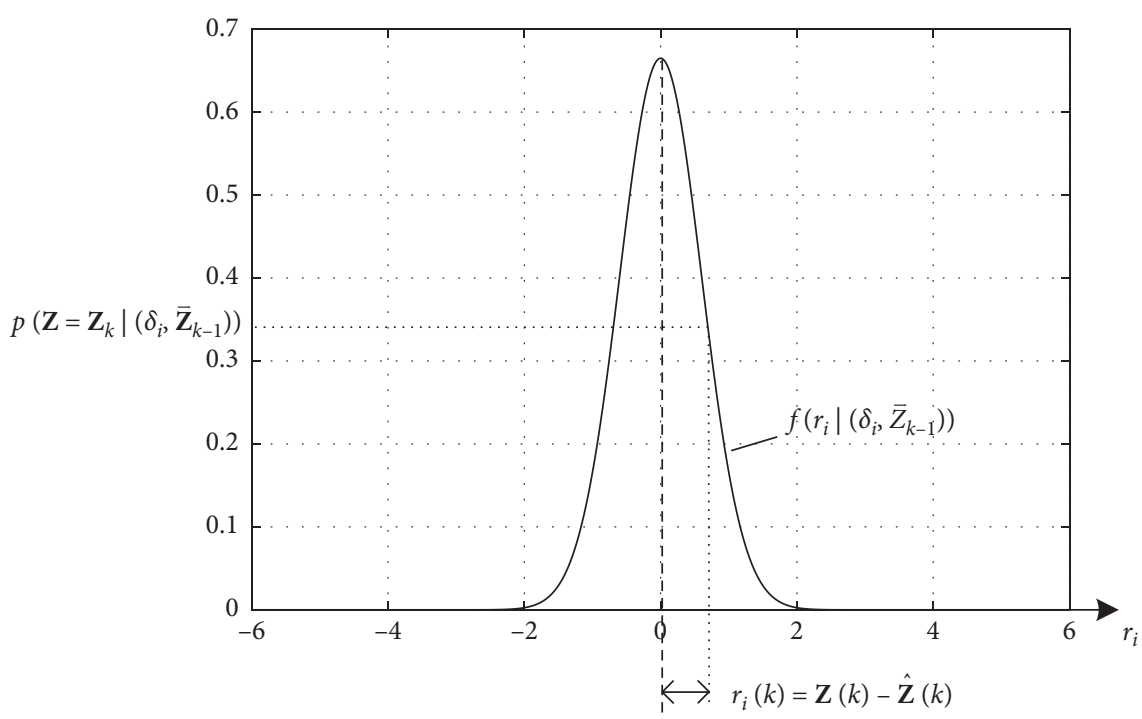

FIGURE 5: Conditional probability density in SISO problem.

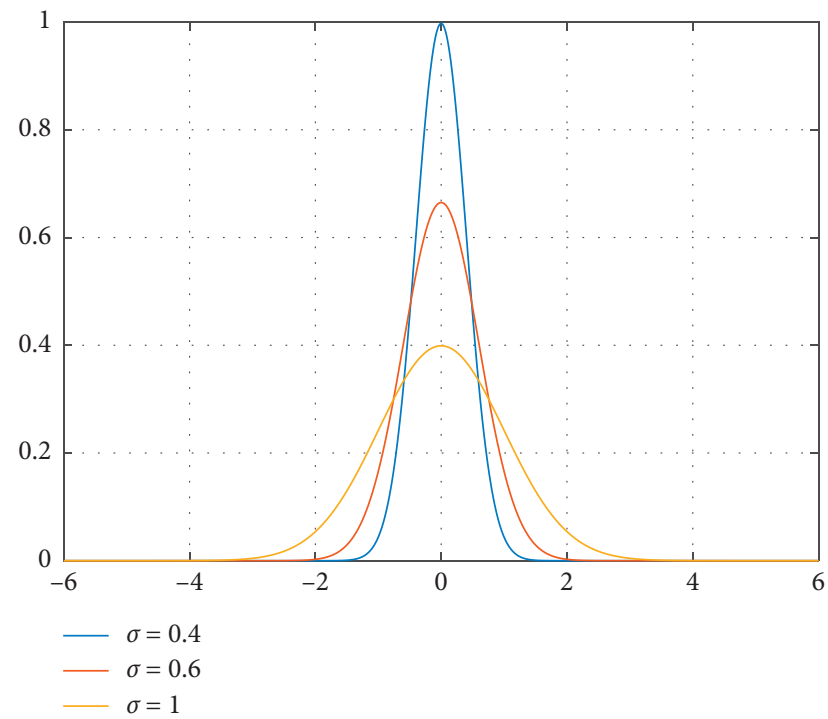

Figure 6: Gaussian functions with different standard deviations.

and its corresponding probability even when an actuator is failure.

$$
\widehat{X}(k)=\sum \widehat{X}_{i}(k) \cdot p_{i}(k) .
$$

\section{Simulation and Result Analysis}

4.1. Simulation Conditions. For the purpose of verifying the effectiveness of the FDD method, simulations of the tiltrotor UAV in the extreme state with the minimum system excitation are carried out with the FDD method discussed in Section 3. For example, in the case of no wind and no maneuver, the tilt-rotor UAV flies in a straight line with constant velocity and altitude. At the same time, setting the actuator fault and locking the actuator near the trim angle, the actuator fault is more difficult to detect currently.
In order to obtain the real simulation results, the zeromean white Gaussian noise is used to degrade the measurement data of the sensor to correspond to the typical characteristics of the low-cost sensor, where the standard deviations of the angular rate sensors are selected as $\sigma_{p, q, r}=0.052 \mathrm{rad} / \mathrm{s}$, and the corresponding noise variances are selected as $\sum_{p}=\sum_{q}=\sum_{r}=0.0027 \times I_{3} \mathrm{rad}^{2} / \mathrm{s}^{2}$.

The structure of tilt-rotor UAV is shown in Figure 1. According to the structure, the actuator FDD method should be composed of three extended Kalman filters, including a no-fault extended Kalman filter and two extended Kalman filters corresponding to left and right aileron faults, respectively. Using the six degrees of freedom model of the aircraft, the simulations are carried out in the MATLAB environment.

The tilt-rotor UAV is set to fly in a horizontal straight line at a constant speed of $20 \mathrm{~m} / \mathrm{s}$. During the flight, the left and right ailerons are used to provide the pitch and roll control moment of the tilt-rotor UAV. The actuator fault conditions are set as Figure 7 shows. During flight time $t=0 \sim 300 \mathrm{~s}$, the following faults occur:

(1) $t=60 \sim 100 \mathrm{~s}$, the left aileron actuator is stuck near the zero position

(2) $t=200 \sim 240 \mathrm{~s}$, the right aileron actuator is stuck near the zero position

For the rest of the time, the tilt-rotor UAV flies normally without any fault. Each actuator is assumed to have five possible fault states. Based on the 6-DOF model of the tiltrotor UAV, the proposed FDD method and the MMAE method are used to carry out fault diagnosis simulations separately.

4.2. Simulation Results Analysis. Figure 8 shows the results of actuator fault detection and diagnosis method for a series of fault conditions set above. The top figure marked with nofault probability indicates that when the actuator fault 

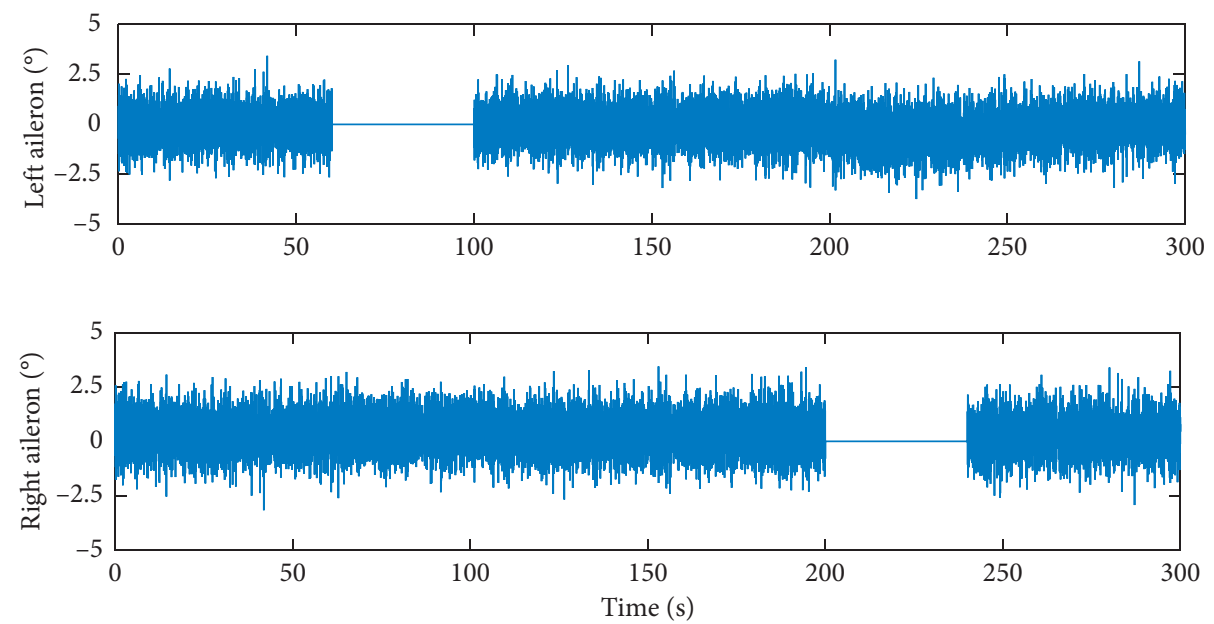

Figure 7: The fault conditions of the left and right ailerons.
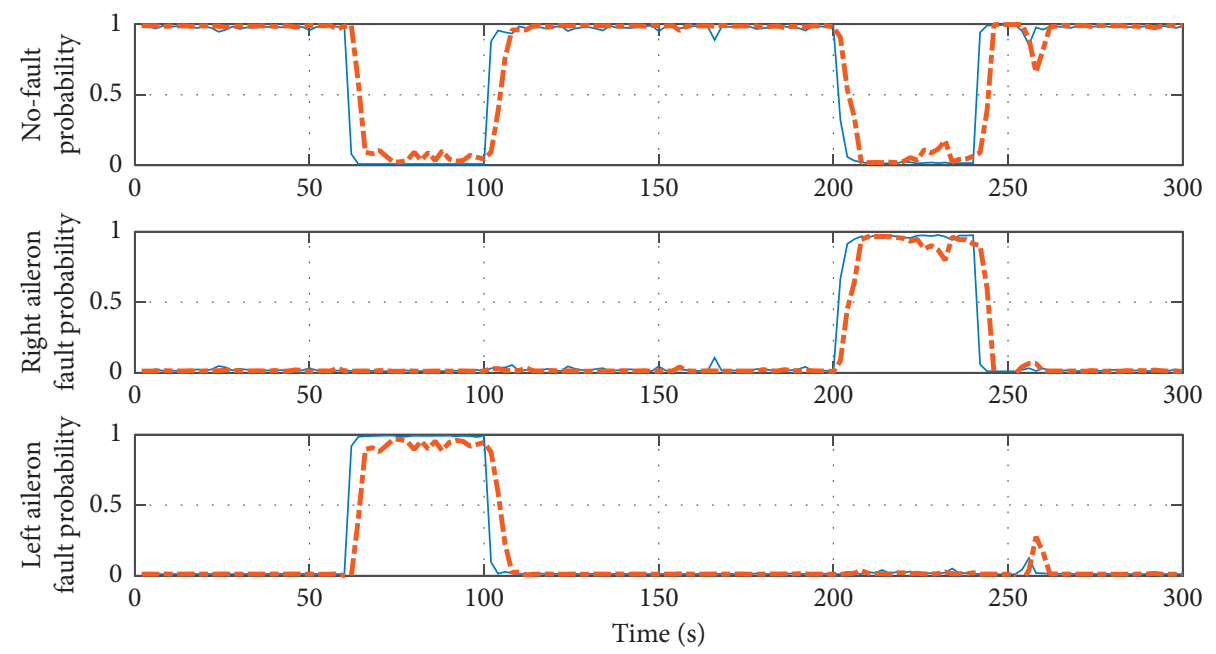

- FDD

-..- MMAE

FIGURE 8: The probability results of actuator fault detection and diagnosis.

detection and diagnosis method does not detect any actuator fault of the tilt-rotor UAV, the no-fault probability is 1 . The middle and the bottom figures indicate the fault probability of the right aileron and the left aileron, respectively. If the fault probability of an actuator is greater than $90 \%$ in a certain period, it means that the actuator has a kind of fault. If the fault probability of an actuator is less than $5 \%$, it means that the fault of actuator has been eliminated. It can be seen from Figure 8 that when the left aileron actuator is stuck near the zero position, the fault probability of the right aileron increases close to 1 according to the actual fault condition. At the same time, the no-fault probability of the tilt-rotor UAV is reduced near to 0 . After $t=100 \mathrm{~s}$, the right aileron is cleared and the actuator works normally. At this time, the no-fault probability returns close to 1 , and the right aileron fault probability decreases to 0 , which means that the tilt-rotor UAV works normally, and no fault is detected in it. In addition, by comparing the results of the proposed FDD method and the MMAE method, it can be concluded that both the
MMAE method and the proposed FDD method can detect the fault when the left and right ailerons fail, respectively. However, using the MMAE method, the fault detection and diagnosis algorithm generally takes longer to detect or to remove the actuator fault, since the MMAE method uses a total of $5 \times 2+1=11$ Kalman filters (the additional filter corresponds to the no-fault condition) in the fault diagnosis algorithm, while the proposed FDD method only uses $2+1=$ 3 Kalman filters (additional filter corresponding to the nofault condition). The computational complexity of the MMAE method is greater than that of the FDD method. As a result, the timeliness of the fault diagnosis algorithm with MMAE method is reduced. Furthermore, Figure 9 shows the deflection estimation performance of actuator. As shown in Figure 9, the deflection of the actuator can be estimated and will be different from that of other times in case of the actuator failure. Therefore, the actuator failure can be detected by the no-fault probability. When the no-fault probability decreases close to 0 , it indicates that an actuator has a fault, and then the 

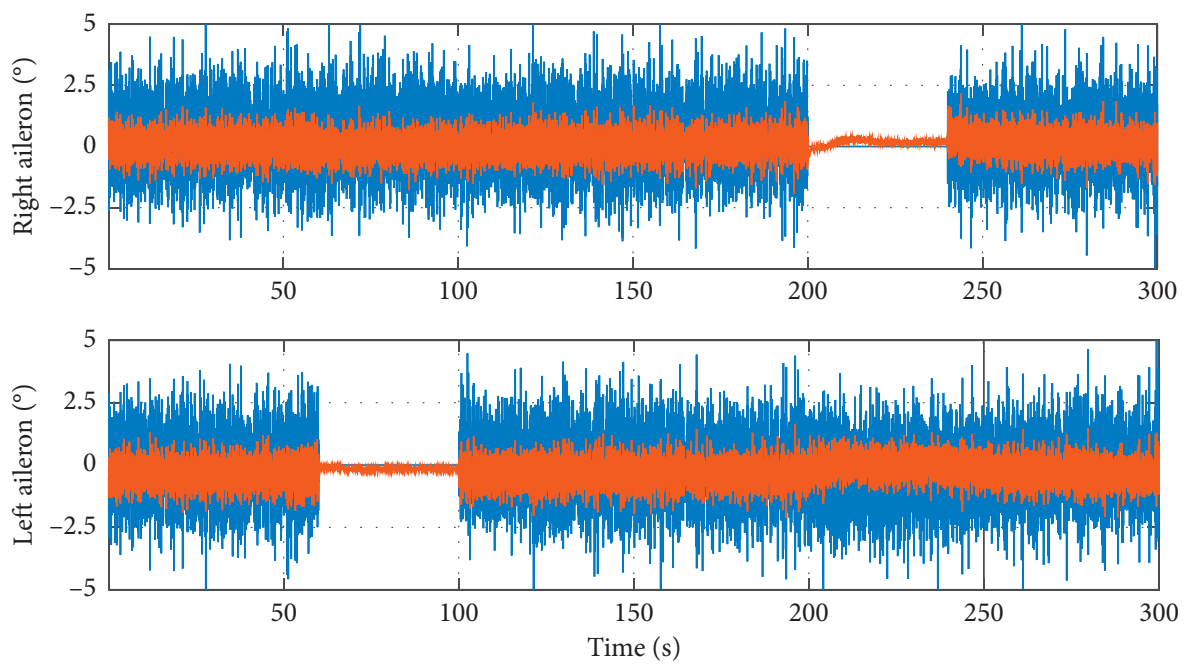

_ True actuator deflection

_ Actuator deflection estimate

FIgURE 9: The comparisons of true actuator deflections and estimations.

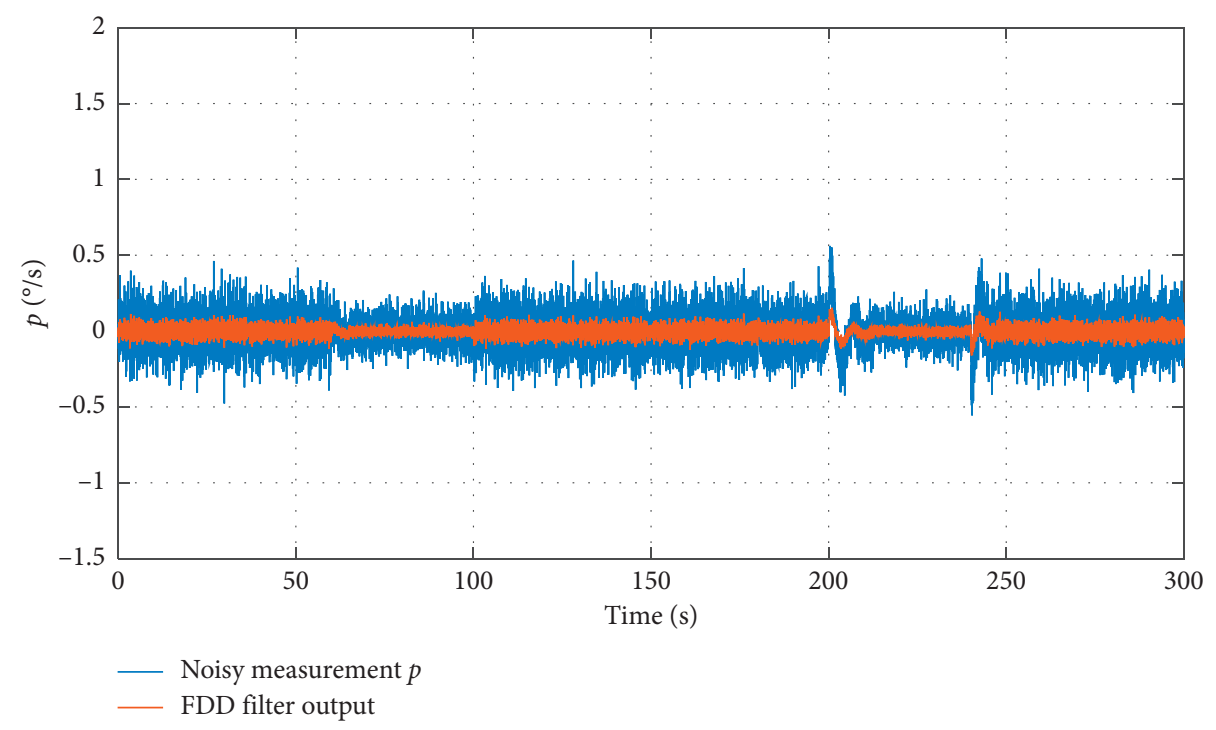

Figure 10: The comparison of measurement $p$ and state variable.

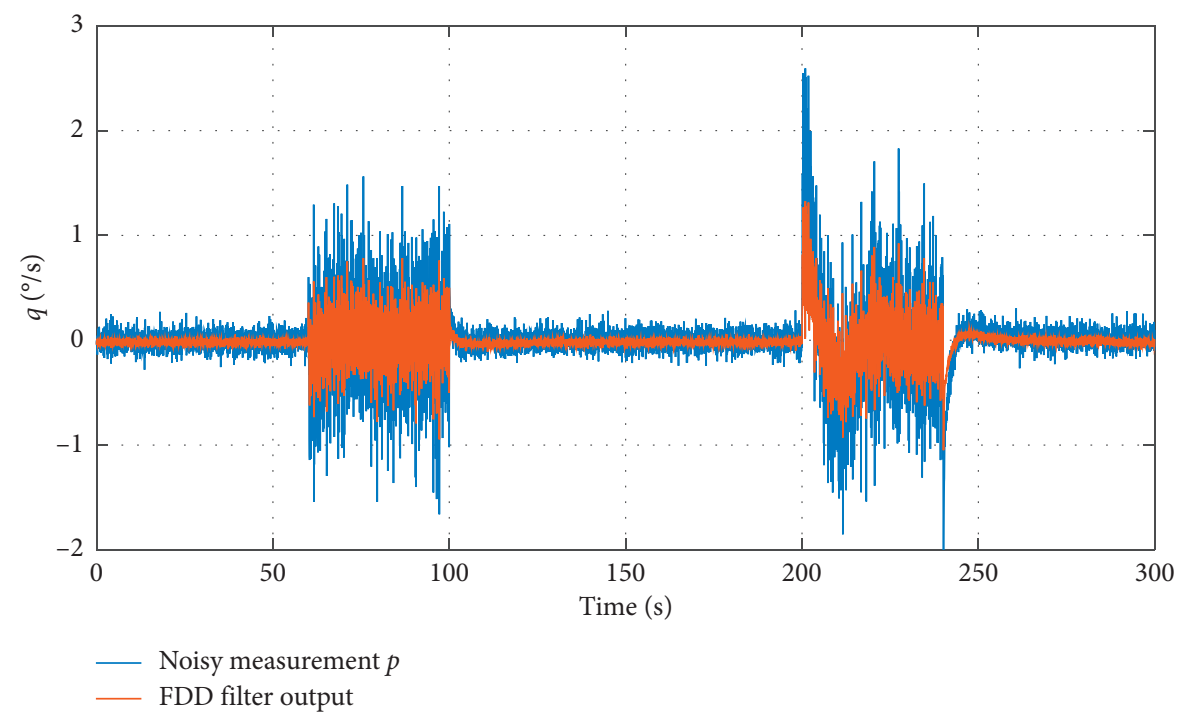

Figure 11: The comparison of measurement $q$ and state variable. 


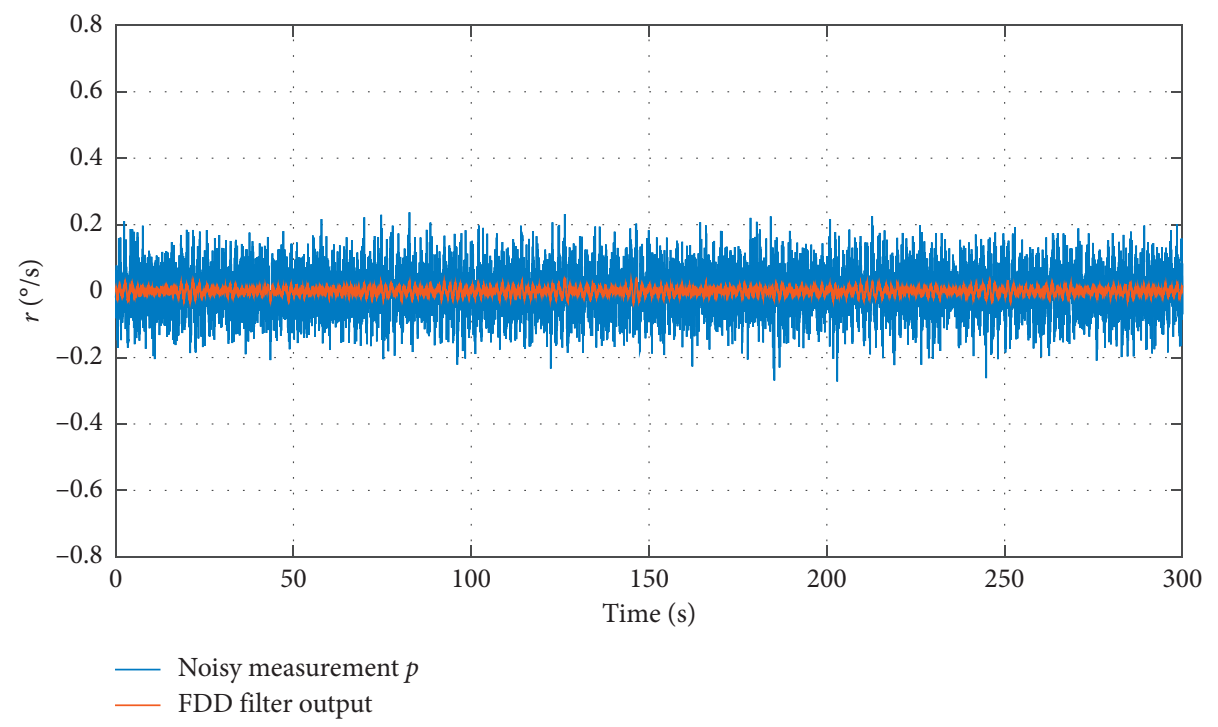

Figure 12: The comparison of measurement $r$ and state variable.

faulty actuator will be detected by judging the fault probabilities of two aileron actuators.

Figures 10-12 show the comparisons between the measurements and the state variables estimated by the FDD method. The blue line is the noisy measurement data, and the red line is the estimated state variables. As shown in the figures, the FDD method also can correctly estimate the state vector even when an actuator is failure.

\section{Conclusions}

The actuator is the implementation of the UAV control system, and it is also an important component part of UAV flight controller. The flight controller needs to control actuators to make the UAV perform various maneuvers and to avoid potential accidents. Therefore, it is very important for tilt-rotor UAVs that the actuator can work stably and reliably. This paper has presented a FDD method for detecting actuator fault of the tilt-rotor UAV. The proposed FDD method is based on the EKF observer, which can be used to estimate the state variables of nonlinear system and to calculate the residual sequences caused by different actuator faults. Then, an actuator fault diagnosis algorithm uses the residuals from each EKF to assign a conditional probability to each fault hypothesis and the complex relationship between actuator faults and observation residuals can be described. Several simulations with the FDD method and the MMAE method have been implemented on a 6-DOF model of the tilt-rotor UAV. The results show that the FDD method can detect the actuator faults successfully with high levels of accuracy and efficiency without any additional sensors to measure actuator deflections or to change the flight control system.

\section{Data Availability}

The airplane design data used to support the findings of this study have not been made available because the project involves intellectual property rights.

\section{Conflicts of Interest}

The authors declare that there are no conflicts of interest regarding the publication of this paper.

\section{Acknowledgments}

The authors would like to acknowledge all colleagues who helped with this research and gave many suggestions.

\section{References}

[1] A. B. Chowdhury, A. Kulhare, and G. Raina, "Back-stepping control strategy for stabilization of a Tilt-rotor UAV," in Proceedings of the Chinese Control \& Decision Conference, Taiyuan, China, May 2012.

[2] A. B. Chowdhury, A. Kulhare, and G. Raina, "A generalized control method for a Tilt-rotor UAV stabilization," in Proceedings of the IEEE International Conference on Cyber Technology in Automation, Control, and Intelligent Systems (CYBER), Khlong Toei, Bangkok, Thailand, May 2012.

[3] H. Chen, X. Zuo, and Y. Zhang, "Tiltrotor aircraft key technology developing research," Flight Dynamics, vol. 25, pp. 5-8, 2007.

[4] G. Wang and Z. Wu, "Configurations and control strategy for VTOL UAVs," Aircraft Design, vol. 3, pp. 25-30, 2006.

[5] F.-J. Lin, Y.-C. Hung, J.-C. Hwang, and M.-T. Tsai, "Faulttolerant control of a six-phase motor drive system using a takagi-sugeno-kang type fuzzy neural network with asymmetric membership function," IEEE Transactions on Power Electronics, vol. 28, no. 7, pp. 3557-3572, 2013.

[6] O. Spinka, O. Holub, and Z. Hanzalek, "Low-cost reconfigurable control system for small UAVs," IEEE Transactions on Industrial Electronics, vol. 58, no. 3, pp. 880-889, 2011.

[7] A. Abbaspour, P. Aboutalebi, K. K. Yen, and A. Sargolzaei, "Neural adaptive observer-based sensor and actuator fault detection in nonlinear systems: Application in UAV," ISA Transactions, vol. 67, pp. 317-329, 2017.

[8] S. Islam, P. X. Liu, and A. El Saddik, "Robust control of fourrotor unmanned aerial vehicle with disturbance uncertainty," 
IEEE Transactions on Industrial Electronics, vol. 62, no. 3, pp. 1563-1571, 2015.

[9] Q. Y. Xia, J. F. Xu, and K. B. Jin, "Tilt-rotor aircraft modeling and its manipulation assignment strategy," Journal of Aerospace Power, vol. 28, pp. 2016-2028, 2013.

[10] J. X. Chen and B. Liang, "Control of tilt rotor unmanned aerial vehicle during mode transition," Tactical Missile Technology, vol. 2, pp. 70-76, 2015.

[11] G. Heredia, A. Ollero, M. Bejar, and R. Mahtani, "Sensor and actuator fault detection in small autonomous helicopters," Mechatronics, vol. 18, no. 2, pp. 90-99, 2008.

[12] T. Patterson, S. McClean, P. Morrow, G. Parr, and C. Luo, "Timely autonomous identification of UAV safe landing zones," Image and Vision Computing, vol. 32, no. 9, pp. 568-578, 2014.

[13] H. Duan and Q. Zhang, "Visual measurement in simulation environment for vision-based UAV autonomous aerial refueling," IEEE Transactions on Instrumentation \& Measurement, vol. 64, no. 9, pp. 2468-2480, 2015.

[14] N. Meskin and K. Khorasani, "Actuator fault detection and isolation for a network of unmanned vehicles," IEEE Transactions on Automatic Control, vol. 54, no. 4, pp. 835-840, 2009.

[15] W. Chen and J. Jiang, "Fault-tolerant control against stuck actuator faults," IEE Proceedings-Control Theory and Applications, vol. 152, no. 2, pp. 138-146, 2015.

[16] Y. Zhong, Y. Zhang, W. Zhang, J. Zuo, and H. Zhan, "Robust actuator fault detection and diagnosis for a quadrotor UAV with external disturbances," IEEE Access, vol. 6, pp. 4816948180, 2018.

[17] B. Jiang and F. N. Chowdhury, "Fault estimation and accommodation for linear MIMO discrete-time systems," IEEE Transactions on Control Systems Technology, vol. 13, no. 3, pp. 493-499, 2005.

[18] M. H. Amoozgar, A. Chamseddine, and Y. Zhang, "Experimental test of a two-stage Kalman filter for actuator fault detection and diagnosis of an unmanned quadrotor helicopter," Journal of Intelligent \& Robotic Systems, vol. 70, no. 1-4, pp. 107-117, 2013.

[19] G. Heredia and A. Ollero, "Sensor fault detection in small autonomous helicopters using observer/Kalman filter identification," in Proceedings of the IEEE International Conference on Mechatronics (ICM), Changchun, China, August 2009.

[20] Q. Zhang, X. Wang, X. Xiao, and C. Pei, "Design of a fault detection and diagnose system for intelligent unmanned aerial vehicle navigation system," Proceedings of the Institution of Mechanical Engineers, Part C: Journal of Mechanical Engineering Science, vol. 233, no. 6, pp. 2170-2176, 2019.

[21] P. Eide and P. Maybeck, "An MMAE failure detection system for the F-16," IEEE Transactions on Aerospace and Electronic Systems, vol. 32, no. 3, pp. 1125-1136, 1996.

[22] R. Isermann, Fault-Diagnosis Systems: An Introduction from Fault Detection to Fault Tolerance, Springer-Verlag, Berlin, Germany, 2006.

[23] P. Zarchan and H. Musoff, "Fundamentals of Kalman filtering: A practical approach," Progress in Astronautics and Aeronautics, Elsevier, Amsterdam, Netherlands, 2nd edition, 2005.

[24] R. Wang, C. Zhao, Y. Bai, W. Du, and J. Wang, "An actuator fault detection and reconstruction scheme for hex-rotor unmanned aerial vehicle," IEEE Access, vol. 7, pp. 9393793951, 2019.

[25] P. S. Maybeck, Stochastic Models, Estimation, and Control, Academic Press, Cambridge, MA, USA, 1979. 